\title{
Fetal foot length throughout gestation: a nomogram
}

\author{
Shripad Hebbar ${ }^{1}$, Kopal S$^{2}$, Prashant Adiga ${ }^{3}$, Lavanya Rai ${ }^{4}$
}

\begin{abstract}
Objective: There are certain situations in obstetric ultrasound such as hydrocephalus, anencephaly, short limb dysplasia, where traditional biometric values cannot be used to assess gestational age. In these situations, we have to use other parameters for the accurate assessment of gestational age. One of the useful parameters is foetal foot length because it is easily accessed and measured easily. Objective of our study was to create a nomogram of fetal foot length $(\mathrm{FtL})$ throughout gestation.
\end{abstract}

Methods: It was a longitudinal prospective study of patients between 16 and 40 weeks of gestation. Inclusion criteria consisted of well-establisheddates (consistent with early ultra sound), singleton, non-anomalous fetuses, and intact amniotic membranes. Sonographic measurements included biparietal diameter (BPD), head circumference (HC), abdominal circumference $(\mathrm{AC})$ and femurlength $(\mathrm{FL})$. For every case, the average of three separate measurements of FtL was used. The mean, percentiles and standard deviations for FtL were calculated at 4 weeks intervals starting from 16th week to 40th week. Pearson's correlation coefficient and associated P-values for the relationships between FtL and other biometric measurements were calculated. The data were compared to anomogram of the FtL generated from previous published studies.

Results: Longitudinal foot length measurements obtained in 50 antenatal women were studied. Nomograms were constructed using Mean \pm 2 SD and Percentiles (5th, 50th and 95th centiles). There was strong correlation between foot length measurements and gestational age and could be linearly predicted (Foot Length $=-14.02+2.3609$ * Gestational Age, $R 2=0.97, P<0.001)$. Foot length had better correlation with gestational age compared to other sonologic parameters such as BPD, HC, AC and FLwith Pearson correlation values of $0.94,0.936,0.951,0.96$, respectively (all $p<0.001$ ).

Conclusions: We constructed a nomogram for fetal foot length at various gestation intervals for the local population. There was a linear relationship and good correlation between foot length and other biometric measurements. These nomograms may be useful to assess gestational age when duration of pregnancy is unknown.

Key words: foot length, nomogram, prenatal ultrasound

\section{Introduction}

Estimation of length of gestation is of critical importance in clinical practice to ensure appropriate management of

\footnotetext{
${ }^{1}$ Additional Professor,

${ }^{2}$ Senior Resident,

${ }^{3}$ Associate Professor,

${ }^{4}$ Professor and Head, Department of Obstetrics and Gynaecology, Kasturba Medical College, MAHE University, Manipal, India.

Correspondence: Shripad Hebbar

E-mail: drshripadhebbar@yahoo.co.in
}

newborns and to distinguish pre-term from term infants. Despite recognition that estimation of GA based on maternal recollection of the LMP (Last Menstrual Period) is fraught with error, it is not generally appreciated that the magnitude and direction of this error vary as a function of LMP estimate. Though early trimester (1014 weeks) ultrasound determinations of the fetal BPD (Biparietal Diameter) can predict expected date of delivery within one week, many pregnant women would not have these early ultrasound examinations in developing countries and majority of the time they report late in gestation.
Thus sonographic determination of gestational age (GA) is becoming increasingly important. The most frequently used biometric parameters for the estimation of gestational age are BPD, HC, AC and FL. As pregnancy advances, ultrasound biometric values lose their capability to estimate gestational age accurately especially in preterm and post term pregnancies. These systematic errors in menstrual GA estimates have profound implications for unnecessary induction, dysfunctional labor and cesarean section, and resultant neonatal and maternal morbidity.

The traditional biometric parameters have some limitations, for example, conditions affecting shape of skull (cranial malformations) will affect BPD and HC, abdominal conditions (hydrops, severe growth restriction) affect AC and FL measurements may be difficult to obtain in deeply engaged breech or may be abnormal in limb dysplasias ${ }^{1}$. This indicates that there is a need for alternative measurement to assess gestational age. The present study focusses on role of foetal foot measurement to assess gestational age and develop-ment of institution specific FtL (Foetal foot length) nomogram.

\section{Methods}

Serial sonographic study between 16-20 to evaluate correlation of gestational age with foetal foot length was carried out at Kasturba Medical College Hospital, Manipal.

Inclusion criteria were singleton pregnancy, starting gestational age of 16 weeks, reliable last menstrual period and dates correlated and confirmed by comparison with first trimmest CRL (Crown Rump Length). Once initial criteria were met, those who were subsequently diagnosed to have aberrant foetal growth (due to conditions such as hypertensive 
disorders, gestational diabetes, placental insufficiency) were excluded from the study, as they would give altered ultrasound parameters. Only those patients who delivered between 37-

40 weeks were included in the study as we wanted longitudinal data till term. The final study subjects were 50 low risk pregnant populations who underwent serial scans at 4 weeks

\section{Figure 1. Plantar view of foetal foot and measurement taken from kin edge overlying the calcaneus to the distal end of the second toe (cursers).}

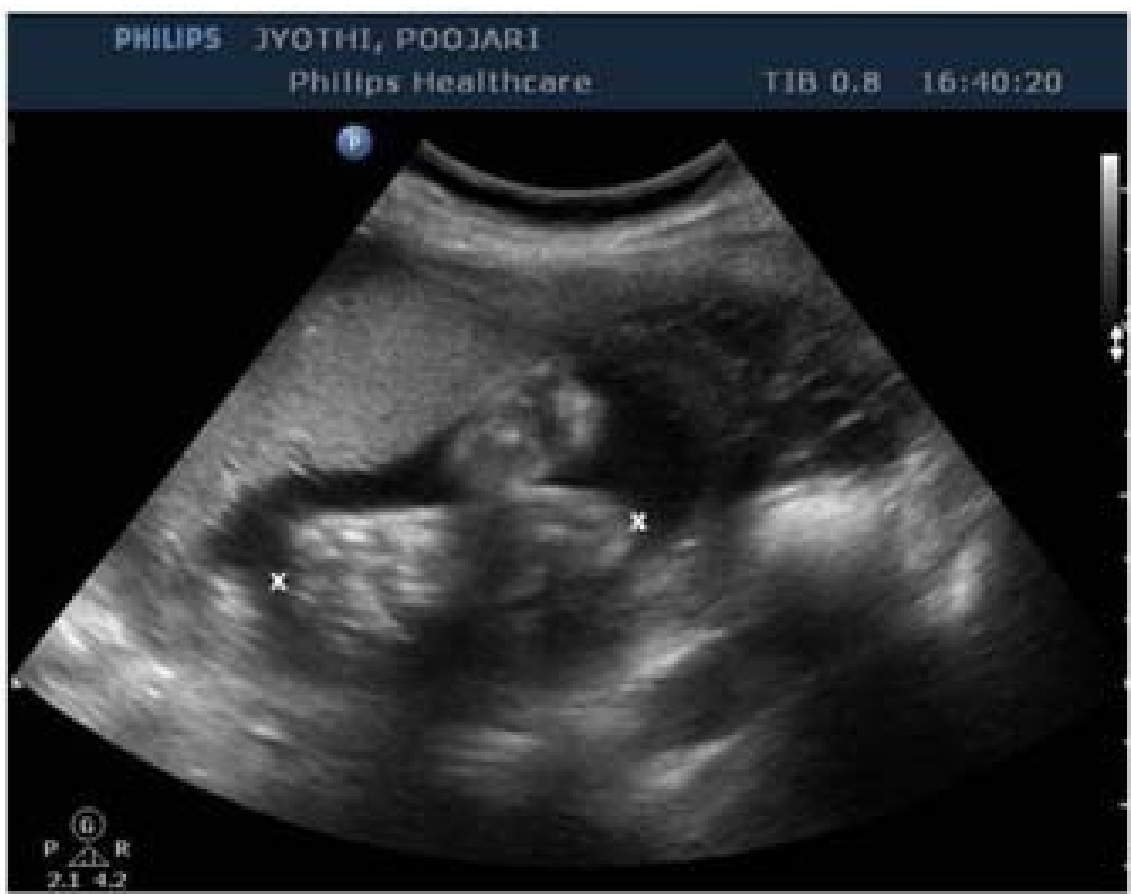

Figure 2. Sagital view of foetal foot and measurement (arrow).

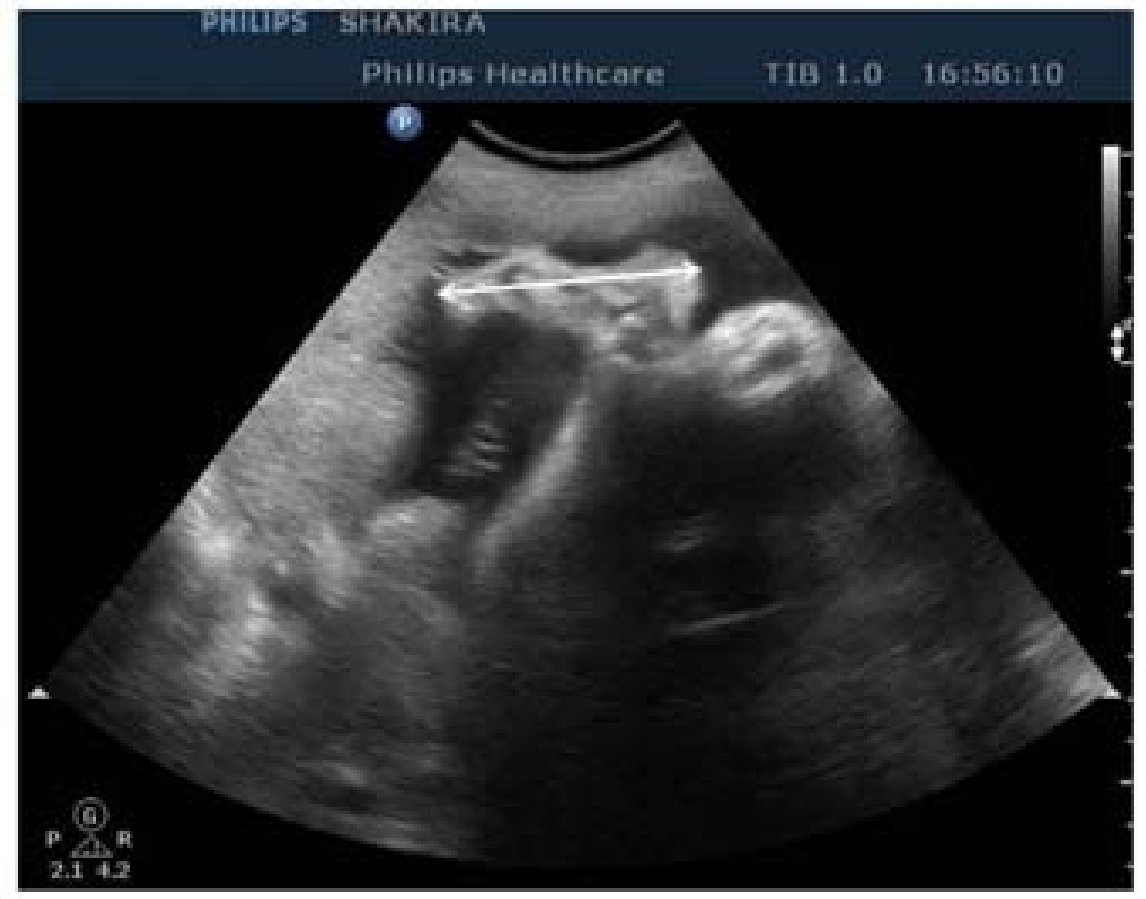

interval starting from 16 weeks till term. Institutional ethical committee approval was obtained for performing this study.

The subjects recruited underwent thorough ultrasonographic examinations using Toshiba Nemio 20 curvilinear array real time 2-D section of linear display scanner with a $3.5 \mathrm{mHz}$ transducer. The biometric measurements included were BPD, HC, AC and Foot Length (FtL). Fetal foot length was taken from the skin edge overlying the calcaneus to the distal end of the longest toe (the first or second toe) on either the plantar or the sagittal view (Figure 1 and 2) and values were averaged. The statistical analysis was performed by SPSS software version 14.

The relationship between gestational ages in weeks to fetal foot length in millimeters was computed by simple linear regression. For a given ges-tational age, predicted values were obtained for the 5th, 10th, 25th, 50th, 75th, 90th and 95th percentiles to develop a nomogram. Correlation coefficient value of fetal foot length measurement was compared with standard biometric parameters such as BPD, HC, AC and FL.

\section{Results}

The mean age of patients studied was 24.7 years, $36 \%$ of them were primigravidae. The average gestational age at the time of delivery was 38.4 weeks. Complete longitudinal data were available for 50 patients starting from 16 weeks till term for the construction of nomogram. With the use of regression equation, the predicted $\mathrm{FtL}$ in centiles were calculated for each GA (Table 1).

The mean values of FtL at corresponding duration of pregnancy along with 2 standard deviations cutoffs are shown in Table 2.

A linear growth function was observed across gestatitional age (GA) and first degree correlation was found to exist between GA and and Foot length (Foot Length $=-14.02+$ 2.3609 * Gestational Age, R2 $=0.97$, 
Table 1. Percentlles values for foetal foot length measurements from 16 to 40 weeks of pregnancy in $\mathrm{mm}$

\begin{tabular}{lccccccc}
\hline $\begin{array}{l}\text { Gestational } \\
\text { Age }\end{array}$ & 5 & 10 & 25 & 50 & 75 & 90 & 95 \\
\hline 16 wks & 19.31 & 19.73 & 20975 & 23.6 & 25.15 & 26.3 & 26.7 \\
20 wks & 29.475 & 30.1 & 31.425 & 33.2 & 35.45 & 36.69 & 37.2 \\
24 wks & 36.155 & 37.2 & 39.55 & 41.1 & 43.85 & 45.69 & 45.9 \\
28 wks & 48.2 & 48.62 & 50.1 & 52.65 & 54.425 & 56.75 & 58.205 \\
32 wks & 59.855 & 60.41 & 61.575 & 64.25 & 67.1 & 68.64 & 68.945 \\
36 wks & 67.11 & 67.4 & 69.275 & 71.6 & 74.5 & 75.89 & 76.5 \\
40 wks & 73.41 & 73.61 & 74.675 & 77.45 & 80.95 & 83.08 & 83.59 \\
\hline & & & & & & & \\
\hline
\end{tabular}

Table 2. Mean and 2 standard deviation cutoff values for foetal foot length measurements from 16 to 40 weeks of pregnancy in $\mathrm{mm}$

\begin{tabular}{cccc}
\hline Gestational age & -2 St Dev & Mean & +2 St Dev \\
\hline 16 wks & 19.32 & 26.3 & 26.7 \\
20 wks & 29.475 & 36.69 & 37.2 \\
24 wks & 36.155 & 45.69 & 45.9 \\
28 wks & 48.2 & 56.75 & 58.205 \\
32 wks & 59.855 & 68.64 & 68.945 \\
36 wks & 67.11 & 75.89 & 76.5 \\
40 wks & 73.41 & 83.8 & 83.59 \\
\hline
\end{tabular}

$\mathrm{P}<0.001)$. Foot length increased at the rate of $2.5 \mathrm{~mm}$ / week between 16 to 28 weeks and $2.2 \mathrm{~mm} /$ week from 29th week till term. A significant correlation was found between $\mathrm{FtL}$ and other traditional growth parameters (such as BPD, HC, AC and FL with Pearson correlation values of 0.94 , $0.936,0.951,0.96$, respectively (all $\mathrm{p}<0.001)$.

\section{Discussion}

The determination of gestational age is important in obstetrics for management of pregnancy and evaluation of foetal development. Higher perinatal mortality has been reported in patients were expected date of delivery is not known. An error in the gestational age estimation can result in prematurity and postmaturity. Extremes of foetal growth contribute disproportionately to overall perinatal and infant morbidity and mortality.

Among various clinical criteria, Last Menstrual Period preceded by normal cycle, is known to correlate best with the gestational age but not reliable when a woman is not sure about her LMP.

Other biometric parameters for GA assessment are BPD, HC, AC and FL. These parameters have their own limitations as BPD after 26 weeks becomes more related to growth and also unreliable in conditions altering the shape of the skull i.e, in breech presentation and oligoamnios. Similarly femur length is also unreliable in cases of achondroplasia ${ }^{1}$.

Our study shows a clear linear relationship between foetal foot length and gestational age with significant correlation between these parameters. This is in comparison with previous studies which conclude that the ultrasonographic measurement of foot length is a reliable indication of gestational age $\mathrm{ag}^{3,4,5}$. A study by Mandarim-de-Lacerda et $\mathrm{al}^{2}$ presents significant curves of the foetal foot length growth in relation to foetal parameters and concluded that these curves are useful in anatomy, forensic medicine, foetopathology, medical imaging, obstetrics and paediatrics. We have developed a nomogram which is almost comparable to that of Western population (Table 3) 3,4,5,6,7. We therefore recommend that foetal foot length should be considered for evaluation of gestational age; especially when other traditional parameters are not conclusive.

Present study showed linear relation of FtL with gestational age (Graphs 1 and 2) with gestational age. The incremental growth of FtL observed in our study is similar to observations in a Nepalese study ([foot length $(\mathrm{mm})=2.494 \times$ Gestational age (weeks)-15.46], $r=0.970$ and $\mathrm{P}<0.0001)^{8}$.

Meirowitz NB et al (2000) conducted a large scale ultrasound examination of foot length on 5372 singleton foetuses between 15 and 37 weeks' gestation ${ }^{5}$. They observed that even in extremes of growth abnormalities (both intrauterine growth restriction and macrosomia), the foetal foot length were not altered significantly. They attributed this to the fact that the alterations in the soft tissue stores of subcutaneous fat of foot in these conditions are probably attenuated by the relatively unaffected foot bones. They opined that foot length can be a reliable indicator of gestational age even in extremes of foetal growth. 
Table 3. Publihed data on foot lenth values and their comparion with present data

\begin{tabular}{|c|c|c|c|c|c|c|c|}
\hline \multirow{2}{*}{$\begin{array}{c}\text { Gestational } \\
\text { age }\end{array}$} & \multirow{2}{*}{$\begin{array}{c}\text { Mercer } \\
\text { et al } \\
(1987)\end{array}$} & \multirow{2}{*}{$\begin{array}{c}\begin{array}{c}\text { Goldstein } \\
\text { et al } \\
(1988)\end{array} \\
\begin{array}{c}50 \text { th } \\
\% l e\end{array}\end{array}$} & \multirow{2}{*}{$\begin{array}{c}\begin{array}{c}\text { Molly } \\
\text { et al } \\
(1994)\end{array} \\
\begin{array}{c}50 t h \\
\% l e\end{array}\end{array}$} & \multirow{2}{*}{$\begin{array}{c}\text { Meirowitz } \\
\text { et al } \\
(2000)\end{array}$} & \multirow{2}{*}{$\begin{array}{c}\begin{array}{c}\text { Lyn S. } \\
\text { Chitty } \\
(2002)\end{array} \\
\begin{array}{c}50 \text { th } \\
\% l e\end{array}\end{array}$} & \multicolumn{2}{|c|}{ Present study } \\
\hline & & & & & & $\begin{array}{c}\text { Mean } \\
\text { FtL }\end{array}$ & $\begin{array}{l}\text { 50th } \\
\% \text { le }\end{array}$ \\
\hline 16 wks & 21 & 23 & 22 & 21 & 20.6 & 26.3 & 23.6 \\
\hline 20 wks & 33 & 33 & 32 & 32 & 32.6 & 36.7 & 33.2 \\
\hline 24 wks & 44 & 46 & 42 & 45 & 44.6 & 45.7 & 41.1 \\
\hline 28 wks & 58 & 53 & 53 & 56 & 55.3 & 56.8 & 52.7 \\
\hline 32 wks & 63 & 57 & 63 & 65 & 65.1 & 68.6 & 64.3 \\
\hline 36 wks & 74 & - & 73 & 75 & 73.3 & 75.9 & 71.6 \\
\hline 40 wks & 82 & - & 83 & - & 79.6 & 83.1 & 77.5 \\
\hline
\end{tabular}

\section{Graphs 1 and 2}

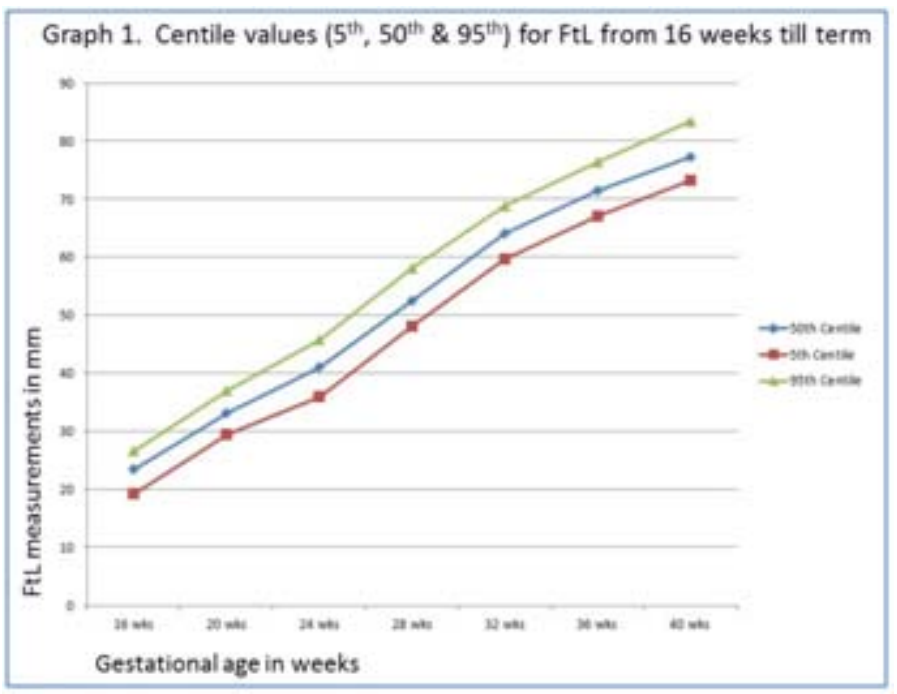

Graph 2. Mean and \pm 2 St Dev values for FtL from 16 weeks till term

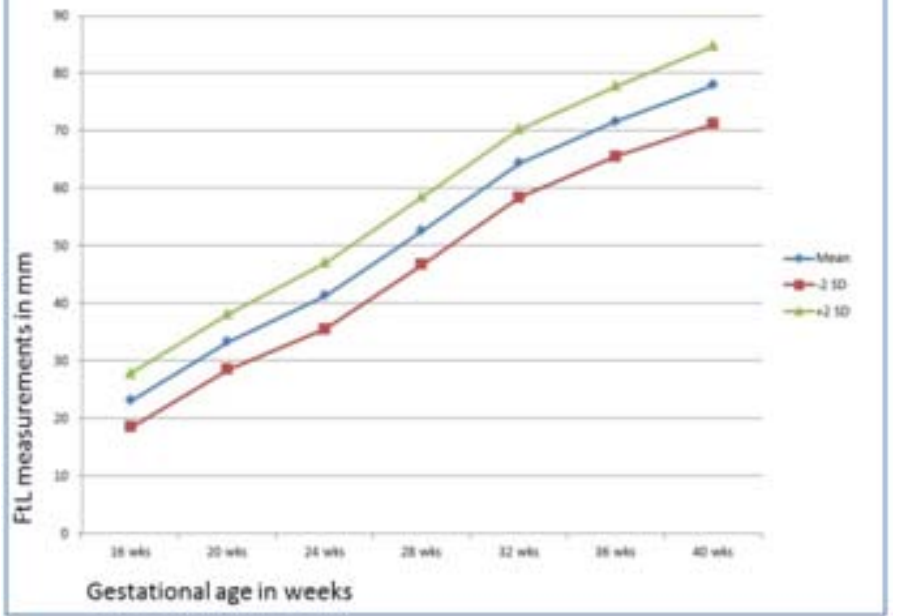

\section{Conclusion}

In normally developing foetus, the FtL increases with advancing gestational age. FtL showed good correlation with gestational age (Pearson's Correlation coefficient $=0.97, \mathrm{P}<0.001)$. So FtL is a good marker for gestational age estimation and can be used in cases of wrong dates. The present data offer the normal range of the FtL measurements throughout the gestation. These values may allow intrauterine assessment of foetal growth.

\section{Acknowledgement}

This study was possible by institutional grant received by Manipal University.

\section{REFERENCES}

1. Mhaskar R, Agarwal N, Takkar D, Buckshee K, Anandalakshmi, Deorari A. Fetal foot length - a new parameter of gestational age. I Int J Gynaecol Obstet, 1989; 29: 35-8.

2. Mandarim-de-Lacerda CA. Foot length growth related to crown-rump length, gestational age and weight in human staged fresh fetuses. An index for anatomical and medical use. Surg Radiol Anat 1990; 12: 103-107.

3. Mercer BM, Sklar S, Shariatmadar A, Gillieson BS, D'Alton ME. Fetal foot length as a predictor of gestational age. Am J Obstet Gynecol 1987; 156(2): 350-5.

4. Goldstein I, Reece EA, Hobbins JC: Sonographic appearance of the fetal heel ossification centers and foot length measurements provide independent markers for gestational age estimation. Am J Obstet Gynecol 1998; 159: 923-6.

5. Meirowitz NB, Ananth CV, Smulian JC, McLean DA, Guzman ER, Vintzileos AM. Foot length in fetuses with abnormal growth. J Ultrasound Med 2000; 19(3): 201-5.

6. Lyn S. Chitty, Douglas G. Altman. Charts of fetal size: limb bones. BJOG 2002; 109: 919-29.

7. Molly S. Chatterjee, Luis A. Izquierdo, Bobby Nevils, George J. Gilson, Cesar Barada. Fetal foot: evaluation of gestational age. 1994. http://www.thefetus.net/

8. K S Joshi, S B Marahatta, S Karki, S Tamrakar, N C Shrestha. Fetal Foot Length and Femur/Foot Length Ratio: Significance in Nepalese context. NJR 2011; 1: 15-22. 